\title{
High-strength concrete with new organic mineral complex admixture
}

\author{
Makhmud Abu-Khasan ${ }^{1}$, Valentina Soloviova ${ }^{1, *}$, and Dmitry Soloviov ${ }^{1}$ \\ ${ }^{1}$ Emperor Alexander I St. Petersburg State Transport University (PGUPS), Moskovsky pr. 9, 190031, \\ St. Petersburg, Russia
}

\begin{abstract}
There are the great problems of the concrete for high voltage the transmission lines obtaining. One of them is the creation of reinforced concrete centrifugally spun transmission towers with enhanced reliability and durability for high-voltage transmission lines having voltage of 110-750 $\mathrm{kV}$. The second problem is the creation of high-quality reinforced concrete foundations to fix overhead electric line lattice towers in the ground because more than $50 \%$ of operated reinforced concrete towers and foundations are in need of repair or were put into repair. A complex high-performance organic mineral admixture for tower bodies of high-voltage transmission lines is developed. The main components of the admixture are polycarboxylate polymere and silica sol. It insures increased compressive strength and tensile strength in bending, freeze resistance, waterproofing and corrosive resistance. The obtained positive results allow to reduce a compression zone width and a crack growth width. The paper can be useful for not only the transport construction but and for concrete obtaining with special properties at any fields.
\end{abstract}

\section{Introduction}

The increase of compressive strength and tensile strength in bending are mainly required for the structures under consideration in order to reduce a crack growth width during the operation of high-voltage transmission line towers and the rise of corrosive resistance and freeze resistance are needed to enhance durability of high-voltage transmission lines' reinforced concrete elements [1-10].

A solution of given problems can be achieved by the maximum use of internal reserves of Portland cement and other components of the system as a result of high-performance chemical activation with the use of a new type of a multifunctional chemical admixture. At the same time the admixture of this kind must ensure efficiency in all these parameters [1116].

The existing and the currently known chemical additives do not ensure the obtainment of concrete with the properties listed above.

To create a new effective admixture, it is advisable to use modern polycarboxylate polymers as the main component. They are able to have a defined and pre-planned effect on the material properties. However, one knows that with all the advantages of polycarboxylate

*Corresponding author: pgups1967@mail.ru 
polymers their efficiency is dependent on various factors including the alkaline environment $[17,18,19]$.

Therefore, in order to stabilize of polymer dispersion and to enhance corrosive resistance of concrete the use of a high molecular weight component is effective. It has dispersing and air-entraining properties and can have a positive effect on the formation of homogeneous finely porous structure of a hardened material.

A modern high-performance surface-active agent represented by a polycarboxylate polymer under the technical name WRM was used as the basis of the created admixture. This polymer has an average side chain length and a good adsorptive power.

\section{The methods and results}

It was experimentally found that the polycarboxylate polymer in an aqueous solution form having $20 \%$ concentration produces an efficient plasticizing effect [the increase of concrete mobility from $\Pi 1$ grade $(2-4 \mathrm{~cm})$ to $\Pi 3$ grade $(12-16 \mathrm{~cm})]$. The rational number of the $20 \%$ aqueous solution is $0,8-1,0$ mass $\%$ of the cement mass.

The silica sol was investigated as the next complex admixture component having excessive reaction activity $[2,5]$. The main characteristics of the silica sol are the following: $\mathrm{pH}=3,5$; density is $1,014 \mathrm{~g} / \mathrm{cm}^{3}$.

The efficiency of the sol was evaluated in the presence of the $20 \%$ aqueous solution of the polycarboxylate polymer according to the change of compressive strength and tensile strength in bending.

It is established that the rational number of the sol is 0,6 mass $\%$ of the $20 \%$ aqueous solution mass of the polycarboxylate polymer and it is found that the sol in the presence of the polycarboxylate polymer most improves tensile strength in bending (by 39\%). Compressive strength is increased by $31 \%$ and density of the concrete is significantly enhanced (by $23 \%$ ) because water absorption of the control mix which has a value of $4,8 \%$ is decreased to a value of $3,7 \%$ that the concrete with the admixture has.

Another component of the developed admixture was a high molecular weight polymer represented by ethoxylatedl cocoalkylamine. It is an optimized mix of cationic and nonionic surfactants which was added in an amount of 0,2-10 mass $\%$ of the polycarboxylate polymer mass.

It is proved that the addition of high-molecular weight compounds of more than 1 mass $\%$ is not effective.

It is experimentally established that the rational number of high-molecular weight compounds is 0,5 mass $\%$ of the $20 \%$ aqueous solution mass of the polycarboxylate polymer.

The obtained mixture of polymers has air-entraining properties as air content in the activated concrete mix is increased and is $3,7 \%$. At the same time the concrete is characterized by an even distribution of fine pores compared with the control mix. Large pores are completely absent. This can have a positive effect on freeze resistance of the concrete.

Besides, compressive strength is increased by $42 \%$ and tensile strength in bending is most enhanced (by 67\%), probably, as a result of the formation of new strong polymer chains together with polycarboxylate polymers.

The complex admixture consisting of the polycarboxylate polymer, the silica sol and the high molecular weight compound is a high-performance additive in which the admixture components enhance efficiency of each component independently. And at the same time increased combined effect is achieved which is to improve compressive strength by $58 \%$ and tensile strength in bending by $88 \%$. Mobility of the concrete is increased from the grade of $\Pi 1(2-4 \mathrm{~cm})$ to the grade of $\Pi 5(20-24 \mathrm{~cm})$. Density of the hardened concrete is enhanced by 
$29 \%$ which is confirmed by the reduction of water absorption to a value equal to $3.4 \%$, while air content in the concrete mix is 3.8-3.9\%.

It is established that the use of the developed admixture ensures the obtainment of the concrete having the actual compressive strength grade of B60 instead of the design grade of B40, as well as an increased value of tensile strength in bending and, as a consequence, improved crack strength of the material as the coefficient of crack strength is increased by $12.5 \%$ and reaches a value of 0,135 . It is a sufficiently large amount especially for highstrength concrete. It exceeds the requirements of GOST 10180.

For the activated concrete of tower bodies in addition to strength index it is necessary to estimate parameters of durability (waterproofing, freeze resistance and corrosive resistance).

It is established that when the developed admixture is used waterproofing and freeze resistance are doubled. But when the consumption of cement is reduced by $24 \%$ and the activated concrete having the design grade of B40 is produced waterproofing and freeze resistance of the activated concrete are by $50 \%$ higher than the values of these parameters in the base concrete.

Corrosive resistance of the activated concrete determined in a $5 \%$ solution of $\mathrm{MgCl} 2$, in a solution of $\mathrm{H} 2 \mathrm{SO} 4$ with $\mathrm{pH}=3$ and in a $5 \%$ solution of $\mathrm{Na} 2 \mathrm{CO} 3$ is increased because the coefficient of corrosive resistance has a value of 0,92-0,94 which is by $19-20 \%$ higher than one in the base concrete. Hence, the activated concrete belongs to a class of concrete with increased chemical resistance.

According to the physical chemical studies it is established that hydration activity of the concrete mix in the presence of the developed admixture is significantly increased because an amount of chemically combined water is enhanced by $37 \%$. This greatly improves compressive strength. At the same time at a temperature of $830^{\circ} \mathrm{C}$ there is an exothermic effect that corresponds to recrystallization of low-basic hydrosilicates which help to raise tensile strength in bending. According to differential thermal studies it is found that hydrolytic lime is completely absent in the activated concrete as at temperatures of $510^{\circ}$ $530^{\circ} \mathrm{C}$ there is no endothermic effect. Probably, $\mathrm{Ca}(\mathrm{OH}) 2$ enters into synthesis reaction with nanodispersions $\mathrm{SiO} 2$ forming insoluble hydrosilicates. This also has a positive effect on corrosive resistance of the concrete [3, 4].

Based on the results of the tests carried out the strength and deformation calculations of reinforced concrete centrifugally spun transmission tower bodies are made. It is proved that crack control and a crack growth width under the action of wind and ice loads are crucial for tower bodies of high-voltage transmission lines.

Cracking of tower bodies is near the ground or in the ground and is estimated by height of compression zone. The smaller the height of compression zone is, the more resistant to cracking concrete is.

The calculations presented in Figure 1 show that when the developed admixture is used the height of compression zone is reduced by $26 \%$ and is $247 \mathrm{~mm}$ relative to the height of compression zone of the base concrete which is $333 \mathrm{~mm}$.

When a design load is $975,0 \mathrm{kN} \cdot \mathrm{m}$ a crack growth width of the activated concrete is reduced by $17 \%$ and is $0,079 \mathrm{~mm}$ relative to a crack growth width of the base concrete which is $0,095 \mathrm{~mm}$.

Before cracking tower bodies can withstand loads by $12 \%$ higher instead of the required design values and before destruction the load carrying ability of tower bodies is increased by $17 \%$ from a value of $1174,8 \mathrm{kN} \cdot \mathrm{m}$ to a value of $1378,4 \mathrm{kN} \cdot \mathrm{m}$. 


\section{Concrete B40}

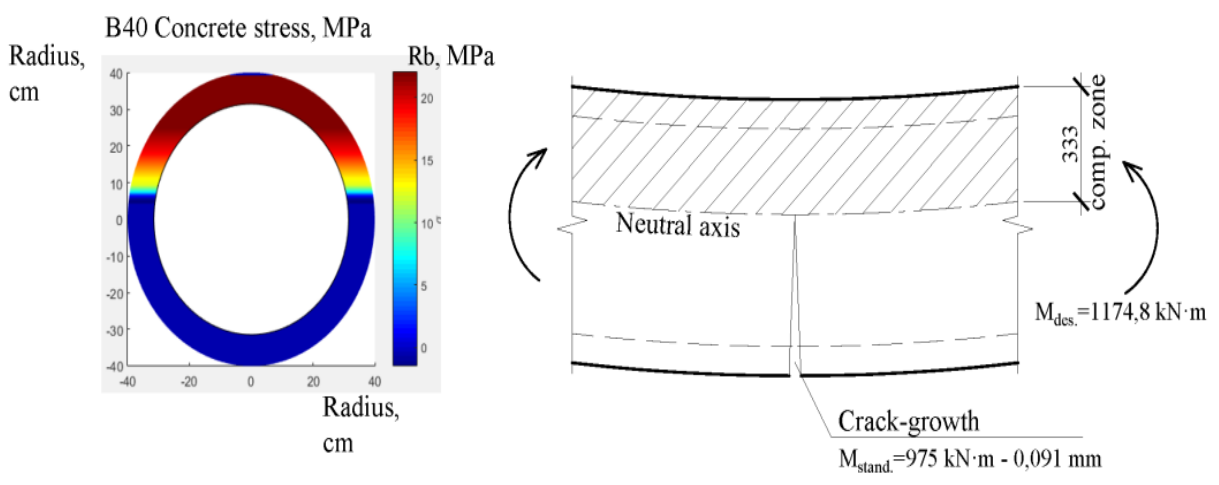

\section{Concrete B60 with the developed admixture}

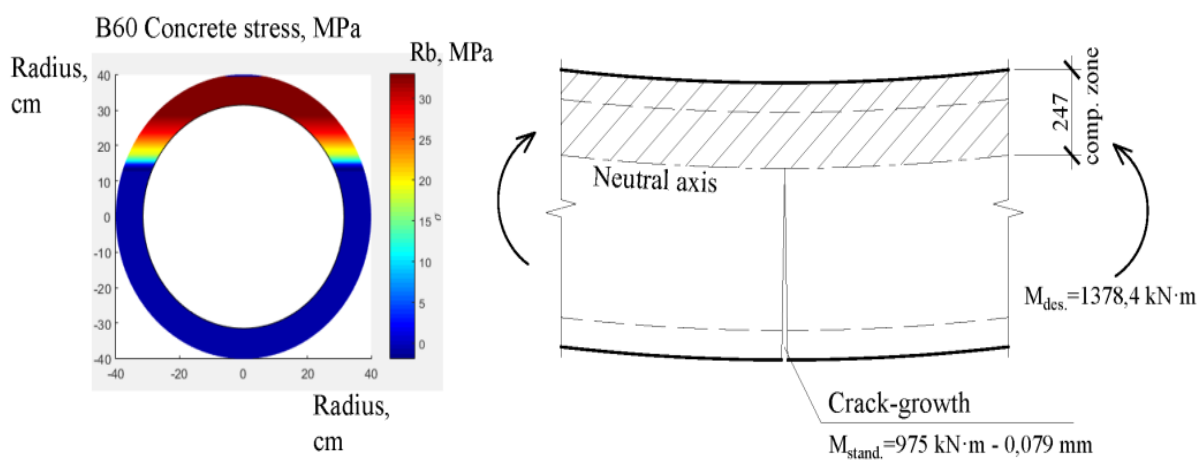

Fig. 1. The results of the reinforced cross-section calculations.

It is found (Fig. 2) that the use of the activated concrete with the actual compressive strength concrete grade of B60 allows to reduce the consumption of reinforcement by $22 \%$ : from $55,6 \mathrm{~kg} / \mathrm{m}$ to $43,5 \mathrm{~kg} / \mathrm{m}$ or in case of the same reinforcing to reduce the consumption of cement by $24 \%$.

Thus, concrete durability is generally increased more than 2 times and is over 100 years instead of 50 year design lifetime as a result of enhancing waterproofing, freeze resistance and corrosion resistance of the activated concrete.

Such kind of results are because of new additive effect. From the fundamental point of view the processes can be explained by means of the papers [20-23].

In the concrete we have a surface with donors and acceptors centers and reactions are started on such kind of centers, because of the chemical analogies silicate, carbons and silica sol. 


\section{Concrete B40, reinforcement stresses, MPa}
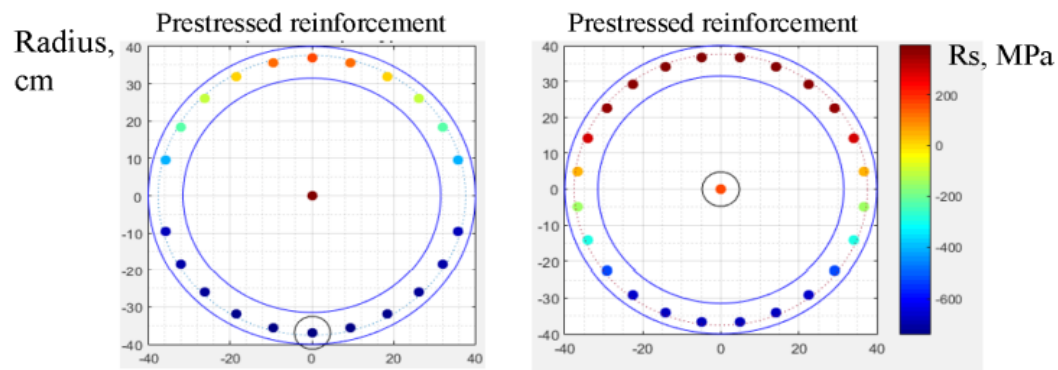

The peak strain of prestressed reinforcement bar is $46,4 \%$ of the standard value. Stress in B40 concrete is $22 \mathrm{MPa}$. Strength is exhausted. Cross-section strength limits the concrete.

\section{Concrete B60, reinforcement stresses, MPa}
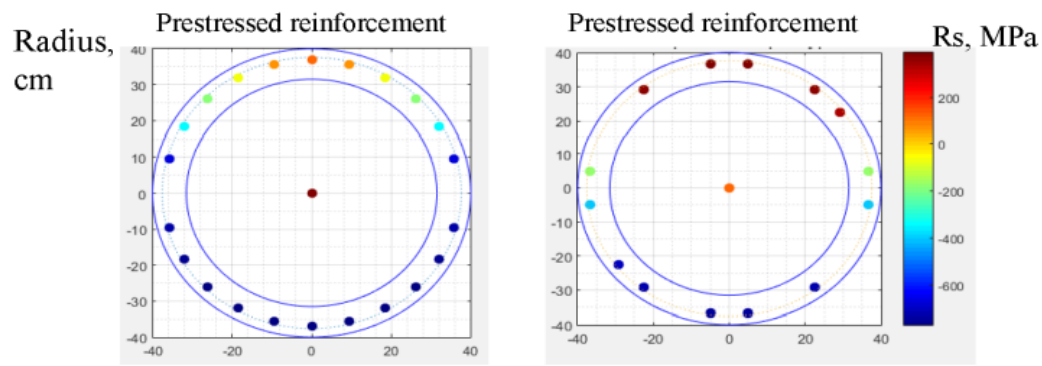

The peak strain of prestressed reinforcement bar is $63,1 \%$ of the standard value. Stress in B60 concrete is $33 \mathrm{MPa}$. Strength is exhausted. Cross-section strength limits the concrete.

Fig. 2. The results of the reinforced cross-section calculations.

\section{Conclusions}

1. New admixture for high strength concrete obtaining is suggested. The main components of the admixture are polycarboxylate polymers and silica sol.

2. Concrete with new admixture has increased compressive strength and tensile strength in bending, freeze resistance, waterproofing and corrosive resistance/

3. The obtained positive results allow to reduce a compression zone width and a crack growth width.

\section{References}

1. L. Maslennikova, M. Abu-Khasan, N. Babak, The use of oil-contaminated crushed stone screenings in construction ceramics, Procedia Engineering, 59-64 (2017)

2. P. Kmokhov, L. Maslennikova, M. Abu-Khasan, Stroitelnye materialy 12, 44 (2003) 
3. L. Maslennikova, L. Svatovskaya, S. Mjakin, I. Vasiljeva, Electron Beam Modification of Solids: Mechanisms, Common Features and Promising Applications (2009)

4. A. Sychova, M. Sychov, E. Rusanova, Procedia Engineering 189, 681-687 (2017) DOI: 10.1016 j.proeng. 2017.05.108

5. A. Sychova, A. Solomahin, A. Hitrov, The Increase of the Durability and Geoprotective Properties of the Railway Subgrade, Procedia Engineering, 189, (688-694) (2017), DOI: 10.1016 j.proeng. 2017.05.109

6. A. Kavetsky, G. Yakubova, M. Sychov, Q. Lin, G. Walter, D. Chan, S. Yousaf, H. Socarras, J. Abrefah, K. Bower, Nuclear Science and Engineering 159, 321-329 (2008)

7. M. Sychov, A.G. Kavetsky, S. Meleshkov, Conversion of radioactive decay energy to electricity, Polymers, Phosphors and Voltaics for Radioisotope Microbatteries (CRC Press, 2002)

8. M. Sychov, Semiconductors 40-9, 1016-1020 (2006)

9. V. Solovyova, M. Abu-Khasan, N. Yershikov, D. Soloviev, E. Kokubin, Innovative technologies in construction and geoecology, Materials of the IV International Scientific and Practical Internet Conference, 17-20 (2017)

10. V. Solovieva, M. Abu-Khasan, S. Kasatkin, N. Yershikov, D. Soloviev, Innovative technologies in construction and geoecology Materials of the IV International Scientific and Practical Internet Conference, 21-25 (2017)

11. V. Solovyova, S. Kasatkin, I. Stepanova, L. Maslennikova, M. Abu-Khasan, N. Yorshikov, Natural and technical sciences 2(104), 146-149 (2017)

12. V. Solovyova, L. Maslennikova, M. Abu-Khasan, I. Stepanova, N. Yorshikov, T.I. Boykova, V. Makarov., S. Kasatkin, Natural and technical sciences 2(104), 150-155 (2017)

13. V. Solovyova, L. Maslennikova, M. Abu-Khasan, et.al., Natural and technical sciences 2(104), 156-162 (2017)

14. L. Svatovskaya, M. Abu-Khasan, E. Rusanova, New technologies for waste management (Saint-Petersburg, 2005)

15. M. Abu-Khasan, Natural and technical sciences 4(36), 259-265 (2008)

16. E. Rusanova, M. Abu-Khasan, Professional education, science and innovation In the XXI century collected works of the XI St. Petersburg Congress, 243-244 (2017)

17. M. Abu-Khasan, E. Rusanova, Natural and technical Sciences 3(105), 58-65 (2017)

18. V. Solovyev, M. Abu-Khasan, N. Ershikov, D. Soloviev, E. Kokubin, Innovative technologies in construction and geoecology, Materials of the IV International Scientific and Practical Internet Conference, 17-20 (2017)

19. V. Solovyova, S. Kasatkin, I. Stepanova, L. Maslennikova, M. Abu-Khasan, N. Ershikov, Natural and technical sciences 2(104), 146-149 (2017)

20. V. Solovyeva, L. Maslennikova, M. Abu-Khasan, I. Stepanova, N. Ershikov, T. Boykova, V. Makarov, S. Kasatkin, Natural and technical sciences 2(104), 150-155 (2017)

21. M. Abu-Khasan, E. Rusanova, Natural and technical sciences 3(105), 58-65 (2017)

22. L. Svatovskaya, L. Lukina, I. Stepanova, et.al. Modern directions of innovative development in materials science. Nanosystems (St. Petersburg, 2016)

23. L. Maslennikova, V. Solovyova, I. Stepanova, et.al., Features of the processes of artificial stone formation and raw materials in obtaining materials (St. Petersburg, 2016) 